\title{
3 Research Square

\section{Evaluation of Visual and Cytology Screening in Preventing Cervical Intraepithelial Neoplasia and Cervical Cancer in Namibia}

Riana Pick

Namibia University of Science and Technology

Koffi Kouame

Walter Sisulu University - Mthatha Campus: Walter Sisulu University

Sylvester Rodgers Moyo

Namibia University of Science and Technology

Yapo Guillaume Aboua ( $\nabla$ yaboua@nust.na )

Namibia University of Science and Technology https://orcid.org/0000-0001-7725-5536

\section{Research Article}

Keywords: Cervical cancer, Cervical intraepithelial lesions, Cryotherapy, Diagnostic outcomes, Human papillomavirus, Screening, Visual screening with acetic acid

Posted Date: October 28th, 2021

DOI: https://doi.org/10.21203/rs.3.rs-1021846/v1

License: (9) This work is licensed under a Creative Commons Attribution 4.0 International License.

Read Full License 


\section{Abstract}

An estimated 851340 women in Namibia are at risk of human papilloma viral (HPV) infection that may cause cervical cancer. Visual screening with acetic acid (VIA) and cryotherapy have been proven highly effective. However, data associating with the impact of cervical cancer screening programmes lack. This study evaluated visual and cytology screening in preventing cervical intraepithelial lesions (CIN) and cervical cancer in Namibia.

Findings showed that women with HPV, cervicitis, koilocytosis and atypia are at an increased risk of testing positive for VIA. Older women, particularly with HIV are more likely to be diagnosed with squamous cell carcinoma (SCC). VIA procedure seems to be viable in screening, although colposcopy in patient follow-up in the Namibian context should be revised and used with positive HR-HPV results. Screening modalities incorporating HPV testing for different ages and risk populations (e.g. HIV positive, autoimmune disease) is recommended. Screening uptake can further be improved by interventions that adopt a model which uses previous diagnostic outcomes, women's health objectives, up-to-date knowledge and practice relevant to national context.

\section{Introduction}

Human papillomavirus (HPV) infection has proven to cause several cancers [1 2]including cervical cancer, which is the global leading cause of mortality in women [3].

The WHO projected that by 2030 , more than 443000 women worldwide will die every year from cervical cancer, with about $90 \%$ of the deaths in Sub-Saharan Africa [4]. As the second highest cancer among women worldwide, cancer of the cervix is diagnosed in 500000 new patients and cause 250000 deaths annually. It is the main cause of morbidity and mortality of women from impoverished resource settings in Africa [5]Of these mortalities, more than $90 \%$ are women from low- and middle-income countries (LMICs) who lack access to primary prevention of HPV vaccine, adequate secondary prevention screening and care to prevent development of cervical cancer [6]. Cervical cancer is furthermore exemplified by the dual burden of communicable and non-communicable disease, standard healthcare associated with scarcity, limitations, prevention challenges, treatment deficit, and inadequate awareness of cervical cancer in the general population as well as health providers [7].

Cervical cancer leads second among women in Namibia, following breast cancer [4]. Also, cervical cancer cases that increased by $50 \%$ is the second leading cause of cancer deaths among women in Namibia [4]. Although, VIA and cryotherapy for early detection of cervical cancer have become increasingly accessible [8], structured guidelines developed by evidence-based research in disease screening and surveillance are necessary to help reduce the burden of cervical cancer in Namibia.

There is a need to enhance policies and programmes directed at cervical cancer screening adherence, accurate follow-up, and applicable treatment. Assessing screening models and trends may also ascertain variances correlated with cervical cancer mortality and illuminate patient characteristics attributing to 
response to treatment and consequently clinical outcome. Additionally, identifying areas to monitor and improve cervical cancer prevention programmes, coordinate related health care services; computing patient data surveillance will help to improve diagnosis, advance strategy to treat cancerous lesions and prevent cancer; improve patient adherence and knowledge on disease.

This study provides evidence-based for cervical cancer screening by inference of single and combined methods.

This study aimed to evaluate cervical cytology and visual inspection of the cervix in the diagnosis of CIN and cervical cancer in Namibia by comparing and contrasting diagnostic outcomes of patients using single and combined methods in cervical cancer screening.

\section{Methodology}

This was a retrospective cohort study, conducted at the Obstetrics and Gynaecology department, Windhoek Central Hospital during 2020. Ethical approvals were obtained respectively from the Research Ethics Committees of Namibia University of Science and Technology (FHAS 13/2020) and the Ministry of Health and Social Services (17/3/3 RP) ethics committees respectively.

The study population consisted of 214 cases of women aged 21-65 years, who were screened and treated for HPV associated lesions, precancerous, cancerous lesions, and remedial early-stage cervical cancer in 2020. Cases of women who were diagnosed and treated for advanced and invasive cancer that was chronic and/or terminal did not form part of this study. In addition, women without a cervix, an abnormal cervical mass or who also had a complete hysterectomy as well as other concurrent cancer or serious medical conditions contraindicative to cancer therapy were excluded from the study. Women who had their menstrual period were also excluded, including women above 65 years and younger than 21 years did not form part of study, unless otherwise recommended with motivation.

The study only focused on cases comprised of HPV infection and CIN (including early-stage cancers which can be treated to prevent progressive or untreatable cervical cancer e.g. carcinoma in situ aka CIS), as it evaluated prevention of cervical cancer. Hence, HPV infections and CIN defined the study population. Prevalence sampling was used to determine the sample size for the study -

$n=z 2{ }^{\star} \mathrm{p} *(1-p) / d 2[9]$, in conjunction with the 2019 HPV statistics Report for Namibia

A data sheet was used to record the required information for each case. Data entered were checked for completeness; incomplete data were not computed. The data collected were entered and analysed using Excel, BM SPSS version 27 and MedCalc software 19.2.6.

Statistical analysis performed at a confidence interval of $95 \%$ and a $p$ value of $<0.05$, included Pearson's $\mathrm{X}^{2}$ test to compare screening outcomes for variables in study subjects (i.e. Screening History and frequency, follow-up); T-test to test to compare quantitative variables (i.e. Age, parity); Chi-squared test to 
compare different subject characteristics (based on data available from records i.e. smoker, underlying disease, indication of an STD, clinical presentation); and Linear regression model for multivariate analysis to associate main outcome, positive frequency and subject factors with screening technique(s) (i.e. Results from colposcopy, histological observations, therapy and clinical outcome).

\section{Results}

\section{Cervical screening in detecting and preventing CIN and Cervical Cancer}

Table I presents the frequency of screening modalities in women of different age groups. Out of the 214 recorded participants, $72.43 \%$ (155) and $27.57 \%$ (59) were respectively HIV negative and HIV positive women. Colposcopy was confirmed to be the predominant method (66\%) of all cases, followed by PAP (44\%) which particularly scored higher in routine screening $(21.3 \%)$. Colposcopy was indicated in about $11 \%$ of cases respectively for a follow-up and/or repeat, followed by biopsy with $8 \%$ and PAP indicated less popular $(0.5 \%)$. VIA $(5 \%)$ and Colposcopy $(0.9 \%)$ were popular in women who were screened for the first time ( $6 \%$ of the study population). PAP and biopsy were equally performed in women who were given the opportunity to be screened through outreach screening programmes by the Cancer Association of Namibia in collaboration with Windhoek Central Hospital and Katutura Hospital, with VIA and colposcopy scoring least popular in these programmes.

\section{Screening Associations in women}

Screening-related data in Table II revealed that most LSILs were identified by biopsy; subsequently most CIN I lesions were identified by colposcopy. Colposcopy also detected majority of HSILs, followed by biopsy then PAP. Biopsy was established to be the only screening modality which detected and reported 3 cases with CIS. Additionally, infection (HPV, B. vaginosis, Candida spp., Chlamydia spp. And T. vaginalis) and cervicitis were equally detected by biopsy.

Biopsy also detected most cases with invasion or SCC or suggestion thereof. Suggested invasion or SCC or detected invasion or SCC was equally detected by colposcopy.

\section{Screening outcomes - Initial and Final Diagnosis}

Table III $(a, b)$ illustrates the screening outcomes at initial screening and final screening as documented in hospital records.

Combined screening by PAP and colposcopy detected most HSILs followed by triage screening (PAP, biopsy and colposcopy) which detected most LSILs in women (HIV-negative) (Table III a). 
In Table III (b), combined screening by VIA and colposcopy detected most LSILs; and triage screening by PAP, biopsy and colposcopy detected most HSILs in women who are HIV-positive. Most cases suspected of cancer, invasive cancer, squamous cell carcinoma or carcinoma in situ were observed upon initial diagnosis in HIV-positive women screened by various modalities. Although these cases were observed two-fold upon final diagnosis in women who are HIV-negative.

\section{Screening Heterogeneity}

Observations from Table IV (a) revealed that women aged 30-49 reported the highest parity compared to women younger and older than them, as well as sexual partners. Parity was lower in women 29 years old and younger, followed by women aged 50 and older; similarly, sexual partners reported by these women were also lower. Women who had no children scored the lowest, followed by women who reported a parity of 1 . Furthermore, women in all age populations reported to have at least one sexual partner. Most women reported more than 4 sexual partners.

As with parity and sexual partners in women aged 30-49, most screening were also reported for women in this age range. In particular, single screening modalities in women 40-49 years old scored equal with parity and sexual partners. Data for women aged 30-39 revealed a similar score for parity and screening. Data for women aged 21 or younger scored equal across parity and sexual partners; whereas data for women aged 22-29 scored similar in parity and sexual partners. Women aged 50 or older scored similar for parity, sexual partners and screening.

The T-test (two-tailed distribution and unequal variance due to different means) and the Chi-squared test statistically assessed screening heterogeneity as well as compared case variables and characteristics between cases.

The data and results for parity $(M=3, S D=1.63)$ and sexual partners $(M=2.19, S D=1.15)$ indicate that case variables are similar, $t(154)=0.173, p=.8632586$. In addition, a chi-square test of independence determined the relation between women of different ages and their characteristics (smoking, comorbidities, sexual barriers, single and married). The relation between these variables was significant, $\chi 2$ $(20, N=155)=118.7, p=<.00001$. Subject characteristics are able to distinguish between these cases and associated lifestyle i.e. smoking, infections, use of contraceptives and their marital status.

Observations from Table IV (b) revealed HIV-positive women aged 30-49 reported the highest parity compared to women younger and older than them, as well as sexual partners. Parity was lower in women 29 years old and younger, followed by women aged 50 and older; similarly, sexual partners reported by these women were also lower. Women who had no children scored the lowest, followed by women who reported a parity of 1 to 2 . The frequency of women who reported a parity of 3 and 4 was equal. Furthermore, women aged between 30-49 all reported to have sexual partners (Table IV a, b). Most women reported 3 sexual partners, followed by women with more than 4 sexual partners. Women who reported 4 sexual partners scored the lowest, followed by women with 2 partners. Cases also revealed 
women with one sexual partner were more likely to also just have one child, as these two associations scored equal. As with parity and sexual partners in women aged 30-49, most screening were also reported for women in this age range. In particular, data for women 40-49 years old scored equal for parity and sexual partners, and similar for screening. Data for women aged 30-39 revealed a similar score for parity and screening. Data for women aged 21 or younger scored equal across parity, sexual partners, and screening; whereas data for women aged 22-29 scored equal in parity and screening, and similar for sexual partners. Women aged 50 or older scored similar for parity and screening. The T-test (two-tailed distribution and unequal variance due to different means) and the Chi-squared test was used to statistically assess screening heterogeneity as well as compare case variables and characteristics between cases of women with HIV. The data and results for parity $(M=2.12, S D=1.9)$ and sexual partners $(M=1.56, S D=1.3)$ indicate that variables between cases are parallel, $t(58)=0.38, p=$ .7053334. In addition, a chi-square test of independence was performed to examine the relation between HIV-positive women of different ages and their characteristics (smoking, co-morbidities, sexual barriers, single and married). The relation between these variables were significant, $\chi^{2}(4, N=59)=128.59, p=<$ .00001. Subject characteristics in women with HIV were able to distinguish between cases and associated lifestyle i.e. smoking, co-morbidity (i.e. Peripheral vascular disease), use of contraceptives and their marital status.

\section{Outcomes associated with screening and treatment}

Pearson test in addition to multivariate analysis determined main outcomes associated with various screening modalities (as depicted in figure 1, 2 and 3)). In single screening, it was confirmed that early precancerous lesions (CIN I) were detected in 7 of the 155 women (4.5\%); 18 women developed CIN II (11.6\%); and 16 women presented with CIN III or HSIL (10\%). Additionally, invasion, SCC, CIS or cancer was suggested in 5 women (3\%) as seen in figure 1. For combined screening, CIN I (and LSIL) were observed in 28 women (18\%); whereas CIN II was confirmed in 19 women (12\%); and CIN III (and HSIL) also presented in 19 women (12\%). Moreover, invasion, SCC and CIS (figure 2) were suggested in 2 women (1.3\%) who were screened by combined modality. Triage screening observed in 10 women with CIN I or LSIL (6.5\%), 7 women with CIN II (4.5\%), and 8 women with CIN III or HSIL (5\%). Triage screening also identified 3 cases suggesting SCC or cancer (figure 3 ). Multivariate analysis significantly discriminated single screening in figure 1 by punctuations and/or mosaicism ( $p=.0498)$; and combined screening by ASCUS $(p=.0498)$, ASCUS-L $(p=.032)$ and thermal coagulation or cryotherapy $(p=.0498)$. Similarly, in all three different screening modalities (figure 1, 2 and 3 ), suggested (or confirmed) invasion or invasive SCC or SCC ( $p=.032-.0498)$; and treatment by cone biopsy ( $p=.032-.0498)$ were jointly significant. Subsequently, treatment by LEEP/LOOP $(p=.032-.0498)$ were conjointly significant in combined (figure 2) and triage screening (figure 3). Irregular (or abnormal) epithelium ( $p=.0192-.0498$ ) and invasive cancer (or cancer) ( $p=.032$ - .0498) were jointly significant in single (figure 1 ) and triage screening (figure 3). Numerous dissimilarities exist between women who developed HSIL or suggested invasion, SCC, CIS or cancer and women who did not. However, reference to Pearson's and Chi-square in 
addition to multivariate analysis, variables that significantly associated with invasion (SCC, CIS, or Ca) was having a HSIL, CIN II or CIN III result for Pap and for biopsy or colposcopy result (odds ratio $=0.07$; $95 \% \mathrm{Cl}=0.0005-7.4091)$.

\section{Discussion}

\section{Screening modalities and associated diagnostic outcomes}

Sensitivity of VIA and specificity of PAP in this study was consistent with a study described by [10] which showed that Pap was more specific than VIA (62.3 vs. 5.3\%). Additional findings of the study confirmed that although PAP was more specific than VIA, VIA confirmed a higher sensitivity. There was statistically significant $(p=0.05)$ difference between VIA and Pap in the number of confirmed cases of cervical intraepithelial neoplasia by colposcopy (42 versus 21 ) for VIA and Pap respectively. These findings collectively suggest VIA to be a viable screening tool in public healthcare settings. Colposcopy is extensively employed in LMICs, to diagnose CINs and direct cervical biopsy sites for women with anomalous cytology, infected with HPV, and clinical presentation suggesting cervical diseases [11]. Additionally, [12] highlighted that women who test positive for $\mathrm{p} 16$ are immediately referred for colposcopy. Although in this study, less than $2 \%$ of the cases documented a p16 positivity result. Likewise, colposcopy remains incorporated in primary follow-up in guidelines and is popular for its application in follow-up post-treatment for CIN, although its variability in observation and poor sensitivity is evident. Relatedly, studies which describe the function and supplementary contribution of colposcopy in follow-up are rare. Evidence backing the usefulness of colposcopy in the follow-up even when resection margins remain positive still lack [13]. Reference [13] describe performance of colposcopy post-LLETZ as poor and incoherent in identifying patients that developed recurrent CIN. Mainly, colposcopy proved unviable in patient follow-up post-treatment for $\mathrm{CIN}$ - thus, colposcopy should only be administered for HR-HPV positive patients after treatment [13]. These study outcomes showed that PAP is the principal method in routine screening (22.5\% in HIV-negative cases and $20 \%$ in women with HIV) - this finding is in agreement with a study by [7] where it was shown that screening was frequently performed by PAP (31.6\%). The current study revealed most HSILs were established by colposcopy as well as PAP smear screening; with biopsy identifying most HSILs of CIN II or III, followed by PAP with colposcopy (combined). Women presented with HSIL in cytology as seen in this study are known to be at risk of developing CC. A recent study showed that $60 \%$ of women with HSIL on cytology, are diagnosed with CIN II or higher upon colposcopy [14]. The chi-square test for the study population $(\mathrm{N}=214)$ showed significant difference between different screening modalities $(p=.00001)$.

\section{Heterogeneity between Screening modalities}


Heterogeneity was assessed by Student's T-test and Chi-square in the total population $(N=214)$ to better understand characteristics that have a plausible impact in cervical diagnosis. Characteristics like age, relationship status, number of sex partners and number of children defined the variables used to assess heterogeneity. Case heterogeneity in various screening modalities was distinguished by women who were mostly single, 30 years and older followed by women 50 years and older, multiple sexual partners (on average 11), a high parity (8 children). Sexual behaviours, specifically number of sexual partners is considered a risk. Other findings revealed that these cases are also highly associated with HPV, an HSIL, CIN III and suspicion (or not excluding) of invasive cancer (SCC, CIS, AIS, early-stage cancer). An HSIL represents a competent proxy for an assiduous high-risk HPV infection. Correspondingly, the familiar viral HPV aetiology with regard to cervical tumours rationalises why women with previous CIN II or CIN III are at a significantly increased risk of vulvar and vaginal cancer. Analysis further identified clinical presentation of chronic lower abdominal pain (specifically in older women), indication of contraceptives, smoking and menopause. In fact, [15] describe smoking as the mutual risk factor and most plausible root of correlation between cervical and lung or bladder cancers as well as confirmed a formidable association between oral and cervical HPV related lesions in smoking patients than non-smokers. Data from a study by [16] showed the need to identify post-menopausal women at risk of developing CIN or cancer prior to completion of screening programme. Postmenopausal women represent a distinct screening population, as they are at an increased risk for recurrent HR-HPV infections than younger women. Furthermore, specific HR-HPV types 16, 18 and 31 seems to link with prospective development of early-stage cancer or worse in this particular age group [16].

\section{Analogy of screening modalities in early detection of cervical intraepithelial neoplasia}

Combined screening modalities significantly associated ASC-US and ASCUS-L ( $p=.0343$ and .0218). Application of immunohistochemistry p16 test in screening would complement the HR-HPV test as well as accurately diagnose atypical lesions (ASC-US, ASCUS-L) where an HSIL cannot be excluded [17]. Combined screening modalities significantly associated with more outcomes than single and triage modalities, namely ASC-US $(p=.0343)$ and ASCUS-L $(p=.0218)$. Management by LEEP/LOOP $(p=.0218$, .0343 and .0343$)$ and cone biopsy $(p=.0343, .0477$ and .0218$)$ all significantly correlated with single, combined and triage screening modalities respectively. Management by thermal coagulation or cryotherapy significantly correlated with combined screening $(p=.0477)$. A meta-analysis study in cryotherapy by [18] described cure rates for CIN II- III lesions as $85 \%-92 \%$; while patients with lesions which extended after the cryoprobe or towards the endocervical canal, LEEP was employed. Moreover, 'screen and treat' cervical screening programmes in recent years have increasingly implemented thermal coagulation. Reference [18] further described a meta-analysis study by [19] in a LMIC, which reported an overall response rate of $93-95 \%$ of biopsy confirmed CIN II+ lesions treated by thermal coagulation study concluded it as a safe, viable and efficacious treatment. The rate in women who are HIV-positive 
was lower, underlining the need for meticulous patient follow-up [18]. Reference [18] additionally described a current systematic review and meta-analysis study in thermal coagulation and cryotherapy in LMICs by [20] which reported a parallel response rate in cryotherapy (82.6\%) and thermal ablation (82.4\%), although the study also emphasized the need for additional research specifically in treatment for women with HIV [18]. Further common significant associations drawn from single, combined and triage screening modalities included suggested invasion or invasive squamous cell carcinoma $(p=.0343, .0218$ and .0343); invasive cancer or early-stage cervical cancer $(p=.0218, .0218$ and .0477$)$. Carcinoma in situ $(p=.0477$ and .0343$)$ significantly associated with single and combined modalities. In early stage and/or invasive cancer (i.e. SCC, AIS, CIS), HPV infection particularly by HPV-16, -18 , or both is a crucial key player. Accordingly, HPV-18 is linked with only $8 \%$ of all HSILs (including CIN II, III and invasive-stage cancers) diagnoses (as opposed to HPV-16 which accounts for $46-58 \%$ ), 38-50\% of invasive (AIS, CIS) diagnoses and $50 \%$ of all squamous cell carcinoma and adenocarcinoma diagnoses. Although, cytologic atypia of any degree maybe indicative of invasive cancer. One study found that a ASCUS, ASUC-L, CIN I or LSIL often precedes diagnosis of invasive cancer or carcinoma since these types of cancers frequently concur with squamous dysplasia - $55 \%$ of patients with AIS were found to have an existing CIN. Factors like HIV, immunosuppression, smoking, and hormonal contraceptives pose additional risk for AIS as well as inhibit suppression of HPV. Similarly, the incidence of cervical AIS increased these past few decades, specifically among women in their 30 s and 40 s. Based on preliminary data, the average period for HPV to clear in women with AIS exceeds the clearance time in women with CIN, thus extended surveillance is commended [21].

\section{Conclusion}

The main strengths of the study were: (1) the design allowed evaluation of single, combined and triage screening modalities without influencing clinical outcomes and outcomes of the study. Screening modalities and diagnosis of the study population were established before commencement of study; while the study evaluated association between screening modalities, diagnosis and case characteristics.; (2) the study population allowed the evaluation of various screening combinations and case heterogeneity; (3) the multi-centric nature of the study permitted capturing case dissimilarities (i.e. different towns, backgrounds, socio-economic) revealing plausible challenges in screening and treatment of women with varying cervical conditions. In the study, only one case documented a woman who tested positive for a HR-HPV infection in diagnosis of a CIN III lesion. Currently, cases from hospitals show that public screening programmes lack the required HPV testing. Studies which have addressed HPV testing in screening strategies, has confired its equal cost-effectiveness and viability to that of VIA. HPV testing in conjunction with approaches like VIA, particularly where VIA results may present clinical significant HSILs or a CIN II (especially recurrent) or CIN III, enhances diagnosis, treatment and management of women with potential progressive $\mathrm{CIN}$ at point of care. Such approaches and integration to cervical screening on a national level demonstrate radical change in screening uptake, treatment, incidence and morbidity. It also promotes national reproductive health in women. Hence, general evaluation and surveillance of cancer screening and management programmes are eminent, to better direct and enhance frameworks 
and interventions; establishing health systems that are effective and which address national health objectives and public health [7]. In view of the observations of cases documenting colposcopy and the study findings by [13]; the application of colposcopy in patient follow-up in the Namibian context should be revised and considered to be used only in conjunction with positive HR-HPV results following a treatment. Hence, HR-HPV should be integrated into screening, particularly for suspected HR-HPV lesions or recurrent lesions. In this regard, varying degree of HR-HPV types should perhaps be tabled with effective corresponding screening modalities according to age groups and taking patient cervical history into account.

Combined screening encompassing HPV testing and cytology in post-menopausal women have shown a higher sensitivity than cytological screening alone. Hence, it will be meaningful to evaluate the extent to which HR-HPV mRNA testing is applicable in triage screening in post-menopausal women [16]. Additionally, invasive carcinomas and carcinoma in situ cannot be prevented by cytological screening, neither treat related lesions. Since current evidence in cytological screening to detect carcinoma and invasive-related lesions is limited, HPV testing provides prospective capacity in early detection of such lesions (premature). HPV testing show additional potential to identify women at an increased risk of HRHPV infection that can advance lesions to carcinoma or invasion. Such diagnostic parameters by HPV testing and particularly p 16 positivity should initiate early treatment and advance prevention, which have been explored in mouse model studies. A recent study presented increasing evidence revealing HPV testing shown more effective in declining prevalence of CIN II, III or cancer than VIA and highlighted increasing affordable availability due to cost-effectiveness of rapid HPV test application in HPV-based screening approaches [18]. Consequently, a national VIA mapping healthcare plan may increase cervical screening in Namibian women, making provision for women who lack access to health services as well as treat women with cancerous lesions in time, considering its low-cost. Thoroughly planned and implemented interventions will improve cervical cancer screening uptake; by adopting a model which applies data from previous diagnostic outcomes, prospective women's and public health objectives and up-to-date knowledge and practice relevant to national context. This should include a homogenous, centralised and synchronised programme and database for practitioners or health centres (hospitals, clinics), where patient screening records, treatments, follow-up, outcomes and management is logged accordingly.

\section{Declarations}

\section{Acknowledgements}

We would like to acknowledge the Namibia University of Science and Technology, the Directorate of research \& innovation of Walter Sisulu University. 
Ethics approval and consent to participate

Ethical approval was obtained from the Namibia University of Science and Technology (FHAS 13/2020) and the Ministry of Health and Social Services (17/3/3 RP) ethics committees respectively. The authors declared compliance with all ethical standards.

Consent for publication

The Authors give Consent for publication.

Competing interests

All methods were performed in accordance with the relevant guidelines and regulations.

The authors declare no conflict of interest.

Funding

The study was funded by the Directorate of research \& innovation of Walter Sisulu University.

Authors' contributions

Y.G.A supervised, provided oversight, leadership, editorial comments, and technical input.

Y.G.A, R.P., K.K \& S.R.M. designed the study, developed the methodology.

R.P. conducted the research.

All authors contributed to the manuscript development.

\section{References}

1. Zandberg DP et al (2013) The role of human papillomavirus in nongenital cancers 63(1):57-81

2. Zaravinos AJO, An updated overview of HPV-associated head and neck carcinomas. (2014) 5(12): p. 3956

3. Ferlay $\mathrm{J}$ et al., Cancer incidence and mortality worldwide: sources, methods and major patterns in GLOBOCAN 2012. 2015. 136(5): p. E359-E386

4. Kakehongo N (2018) Factors affecting cervical cancer screening among women of child bearing age, attending Intermediate Hospital Oshakati (IHO), Oshana region, Namibia: a case-control study. University of Namibia

5. Blumenthal PD et al., Training for cervical cancer prevention programs in low-resource settings: Focus on visual inspection with acetic acid and cryotherapy. 2005. 89: p. S30-S37

6. Bertman $\mathrm{V}$ et al., Health worker text messaging for blended learning, peer support, and mentoring in pediatric and adolescent HIV/AIDS care: a case study in Zimbabwe. 2019. 17(1): p. 1-8 
7. Lott BE et al., Interventions to increase uptake of cervical screening in sub-Saharan Africa: a scoping review using the integrated behavioral model. 2020. 20: p. 1-18

8. Castle PE. The new era of primary HPV screening for prevention of invasive cervical cancer. in Cancer Forum. 2014

9. Sinha S et al., Comparing the efficacy of visual inspection of cervix with acetic acid and Lugol's iodine with Pap smear cytology in screening for cancer cervix. 2018. 4(1): p. 10

10. Srivastava A et al., Visual Inspection with Acetic Acid Versus Papanicolaou Test in Cervical Cancer Screening. 2020. 18(3): p. 1-6

11. Xue P, Ng MTA, Qiao YJBm (2020) The challenges of colposcopy for cervical cancer screening in LMICs solutions by artificial intelligence 18:1-7

12. Almonte $\mathrm{M}$ et al., Multicentric study of cervical cancer screening with human papillomavirus testing and assessment of triage methods in Latin America: the ESTAMPA screening study protocol. 2020. 10(5): p. e035796

13. Heinonen A et al., Role of Colposcopy after Treatment for Cervical Intraepithelial Neoplasia. 2020. 12(6): p. 1683

14. Khieu M, Butler SL, High Grade Squamous Intraepithelial Lesion. 2017

15. Preti M et al., Risk of HPV-related extra-cervical cancers in women treated for cervical intraepithelial neoplasia. 2020. 20(1): p. 1-7

16. Asciutto KC, Borgfeldt C, and O.J.B.c. Forslund, 14-type HPV mRNA test in triage of HPV DNA-positive postmenopausal women with normal cytology. 2020. 20(1): p. 1-10

17. Yu L et al., Application of p16/Ki-67 dual-staining cytology in cervical cancers. 2019. 10(12): p. 2654

18. Cubie HA, Campbell CJWsH, Cervical cancer screening-the challenges of complete pathways of care in low-income countries: focus on Malawi. 2020. 16: p. 1745506520914804

19. Randall TC et al., Worthy of further consideration: an updated meta-analysis to address the feasibility, acceptability, safety and efficacy of thermal ablation in the treatment of cervical cancer precursor lesions. 2019. 118: p. 81-91

20. de Fouw $\mathrm{M}$ et al., A systematic review and meta-analysis of thermal coagulation compared with cryotherapy to treat precancerous cervical lesions in low-and middle-income countries. 2019. 147(1): p. $4-18$

21. Teoh D et al., Diagnosis and management of adenocarcinoma in situ: a society of gynecologic oncology evidence-based review and recommendations. 2020. 135(4): p. 869

\section{Tables}

TABLE I: Frequency of Screening modalities $(N=214)$ 


\begin{tabular}{|c|c|c|c|c|c|c|c|c|c|c|c|c|c|}
\hline $\mathrm{Y} / \mathrm{O}$ & B & C & $\mathbf{P}$ & V & $\mathrm{C}+\mathrm{B}$ & $\mathrm{V}+\mathrm{B}$ & $\mathrm{V}+\mathrm{C}$ & & $\mathrm{P}+\mathrm{B}$ & & $\mathrm{P}+\mathrm{C}$ & $P+B+C$ & $\mathrm{~V}+\mathrm{B}+\mathrm{C}$ \\
\hline \multicolumn{14}{|c|}{ HIV negative $(n=155)$} \\
\hline$\leq 21$ & & & 1 & & 1 & & & & & & 1 & & \\
\hline $22-29$ & 1 & 4 & & 11 & & 1 & 5 & & 2 & & 1 & 4 & 2 \\
\hline $30-39$ & 7 & 3 & 4 & & 7 & 1 & 10 & & 7 & & 6 & 10 & 1 \\
\hline $40-49$ & 8 & 6 & 2 & 1 & 3 & 1 & 4 & & 4 & & 9 & 8 & \\
\hline $50-59$ & 4 & & & & 1 & & & & 4 & & 4 & 3 & \\
\hline $60-64$ & & & & & & & & & 1 & & & & \\
\hline$\geq 65$ & 2 & & & & & & & & & & & & \\
\hline \multicolumn{14}{|c|}{ HIV positive $(n=59)$} \\
\hline$\leq 21$ & & & & 1 & & & & & & & 1 & & \\
\hline $22-29$ & & & & & & 7 & & & & & & & \\
\hline $30-39$ & & 3 & & & 1 & 11 & & 1 & & 1 & 6 & 1 & \\
\hline $40-49$ & & & & & & 5 & & 4 & & 3 & 4 & 2 & \\
\hline 50-59 & & & 1 & & & 1 & & 2 & & 2 & 1 & 1 & \\
\hline
\end{tabular}

Years old (Y/O), Biopsy(B), Colposcopy (C), PAP (P), VIA (V).

TABLE II: Screening associations in women

\begin{tabular}{|c|c|c|c|c|c|c|c|c|c|}
\hline 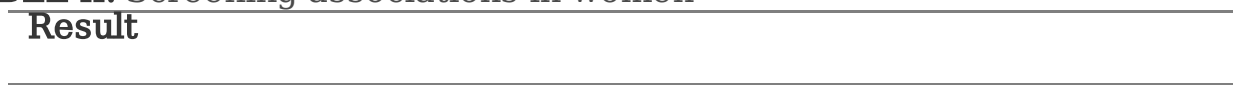 & B & C & $\mathbf{P}$ & V & & & C & $\mathbf{P}$ & V \\
\hline [HIV-ve $\mathrm{n}=155]$ & & & & & & & & & \\
\hline POSITIVE & & & & 25 & 1 & & & & 27 \\
\hline Other (e.g. Mitotic activity/visible SCJ/dysplasia/acetowhitening, etc.) & & 53 & & & & & 41 & & \\
\hline ASC-US & & & 2 & & & & & 2 & \\
\hline ASCUS-L & 1 & & 1 & & & & & 1 & \\
\hline ASC-H & & 1 & 2 & & & & & 3 & \\
\hline LSIL & 14 & 4 & 2 & & & 4 & 7 & 2 & \\
\hline HSIL & 12 & 23 & 11 & 1 & & 6 & 4 & 7 & 2 \\
\hline CIN I & 6 & 15 & 4 & 1 & & 5 & 2 & 4 & \\
\hline CIN II & 19 & 17 & 18 & 7 & & 2 & 3 & 7 & 4 \\
\hline CIN III & 11 & 7 & 9 & 1 & & 2 & 1 & 4 & 3 \\
\hline *CIS & 3 & & & & & & & & 1 \\
\hline *Invasion/ SCC / Cervical cancer & 5 & 3 & & & & 3 & 1 & 1 & \\
\hline Chronic Cervicitis & 21 & 7 & 1 & & & 9 & 2 & & \\
\hline $\begin{array}{l}\text { HPV/BV/ } \\
\text { Candida/Trich }\end{array}$ & 21 & 5 & 9 & & & 6 & 1 & 2 & \\
\hline \multicolumn{10}{|l|}{ Reason } \\
\hline FIRST TIME & & 1 & & 5 & 1 & & 1 & & 6 \\
\hline ROUTINE & & 7 & 35 & 5 & & & 2 & 11 & 6 \\
\hline $\begin{array}{l}\text { PREV. ABN RESULT/ } \\
\text { RE-SCREENING }\end{array}$ & & 2 & 4 & 1 & & & & 2 & \\
\hline FOLLOW-UP/ REPEAT & 11 & 15 & 1 & 3 & & 7 & 8 & 1 & \\
\hline
\end{tabular}

Biopsy (B), Colposcopy (C), PAP(P), VIA (V), HPV test (HPVT), bacterial Vaginalis (BV) Trichomonas (Trich), Squamous columnar junction (SCJ), Atypical squamous cells of undetermined significance (ASC-US), negative (ve), positive(+ve), Low-grade lesions (L), High-grade lesion (H), Squamous intraepithelial lesion (SIL), Cervical intraepithelial (CIN) lesion, dysplasia grade (I/II/III), Squamous cell carcinoma (SCC), Carcinoma in situ (CIS).

TABLE III (a): Detections associated with screening in women 


\begin{tabular}{|c|c|c|c|c|c|c|c|c|}
\hline \multirow[t]{3}{*}{ Screening } & \multicolumn{4}{|c|}{ Initial Diagnosis } & \multicolumn{4}{|c|}{ Final Diagnosis } \\
\hline & \multicolumn{8}{|c|}{ LSIL HSIL*(I)/Ca*Ca/ LSIL HSIL *(I)/Ca/ *Ca/ } \\
\hline & & & $\mathrm{CC}$ & CIS & & & SCC & CIS \\
\hline \multicolumn{9}{|c|}{ HIV-negative $(n=155)$} \\
\hline B & 6 & 4 & 1 & 1 & 10 & 11 & 1 & \\
\hline C & 3 & 10 & & & 3 & 10 & 1 & \\
\hline $\mathbf{P}$ & 5 & 2 & & & 2 & & & \\
\hline V & 4 & & & & 11 & 1 & & \\
\hline$B+C$ & 6 & 5 & 1 & 1 & 5 & 6 & 1 & 1 \\
\hline$P+B$ & 5 & 10 & & & 8 & 6 & 1 & \\
\hline $\mathrm{P}+\mathrm{C}$ & 6 & 16 & & & 4 & 13 & & \\
\hline $\mathrm{P}+\mathrm{B}+\mathrm{C}$ & 13 & 12 & & & 12 & 9 & 1 & \\
\hline $\mathrm{V}+\mathrm{B}$ & 1 & 1 & & & 1 & 1 & & \\
\hline$V+C$ & 6 & 3 & & & 15 & 4 & & \\
\hline $\mathrm{V}+\mathrm{B}+\mathrm{C}$ & 2 & 1 & & & 2 & 1 & & \\
\hline
\end{tabular}

Biopsy (B), Colposcopy (C), PAP(P), VIA (V),

Low-grade squamous intraepithelial lesion (LSIL),

High grade squamous intraepithelial lesion (HSIL)

*- Suspected of/diagnosed with/cannot be excluded

Invasion (I), cancer (Ca), Squamous cell carcinoma (SCC),

Carcinoma in situ (CIS).

TABLE III (b): Detections associated with screening in women

\begin{tabular}{|c|c|c|c|c|c|c|c|c|}
\hline \multirow[t]{3}{*}{ Screening } & \multicolumn{4}{|c|}{ Initial Diagnosis } & \multicolumn{4}{|c|}{ Final Diagnosis } \\
\hline & \multicolumn{8}{|c|}{ LSIL HSIL*(I)/Ca*Ca/ LSIL HSIL *(I)/Ca/ ${ }^{*} \mathrm{Ca}$} \\
\hline & & & SCC & CIS & & & SCC & CIS \\
\hline \multicolumn{9}{|c|}{ HIV-positive $(n=59)$} \\
\hline C & 1 & 1 & & & 3 & & & \\
\hline $\mathbf{P}$ & 1 & & & 1 & & 1 & & \\
\hline$B+C$ & 3 & & 1 & & 2 & 2 & 1 & \\
\hline$P+B$ & 2 & 5 & & & 2 & 3 & & \\
\hline$P+C$ & 2 & 4 & 2 & & 2 & 3 & & \\
\hline$V+B$ & & 1 & & 1 & & 1 & & 1 \\
\hline $\mathrm{V}+\mathrm{C}$ & 11 & 4 & 1 & & 19 & 3 & & \\
\hline$P+B+C$ & 7 & 6 & 1 & & 8 & 2 & 1 & \\
\hline
\end{tabular}

Biopsy (B), Colposcopy (C), PAP(P), VIA (V),

Low-grade squamous intraepithelial lesion (LSIL),

High grade squamous intraepithelial lesion (HSIL)

*- Suspected of/diagnosed with/cannot be excluded

Invasion (I), cancer (Ca), Squamous cell carcinoma (SCC),

Carcinoma in situ (CIS).

TABLE IV (a): Characteristics in study population-ve - negative 


\begin{tabular}{|c|c|c|c|c|c|c|c|}
\hline \multirow{2}{*}{$\begin{array}{l}\text { HIV-ve }(n=155) \\
\text { PARITY }\end{array}$} & \multicolumn{7}{|c|}{$\leq 2122-2930-3940-4950-5960-64 \geq 65$} \\
\hline & & & & & & & \\
\hline 0 & 2 & \multicolumn{6}{|c|}{2} \\
\hline 1 & & 3 & 1 & 2 & & & \\
\hline 2 & 5 & 2 & 10 & 2 & 1 & & \\
\hline 3 & & 2 & 3 & 6 & & & \\
\hline$>3$ & 1 & & 6 & 7 & 3 & 1 & 2 \\
\hline \multicolumn{8}{|c|}{ SEXUAL PARTNERS } \\
\hline 0 & & 1 & & & & & \\
\hline 1 & 1 & 2 & 1 & 5 & 1 & 1 & 1 \\
\hline 2 & 2 & 1 & 2 & 1 & & & \\
\hline 3 & & 1 & 5 & 4 & & & \\
\hline 4 & 2 & 2 & 3 & 3 & 1 & & \\
\hline$>4$ & 3 & 1 & 8 & 4 & 1 & & \\
\hline SMOKING & & & 1 & 2 & & & \\
\hline Co-morbidity & 1 & 1 & 15 & 12 & 4 & & \\
\hline Sexual Barriers & 2 & 3 & 6 & 2 & & & \\
\hline SINGLE & 5 & 6 & 16 & 8 & 2 & & 1 \\
\hline MARRIED & 1 & 1 & 2 & 9 & 2 & 1 & 1 \\
\hline
\end{tabular}

TABLE IV (b): Characteristics in study population +ve - positive

\begin{tabular}{llllll}
\hline HIV +ve $(n=59)$ & $\leq 21$ & $22-29$ & $30-39$ & $40-49$ & $50-59$
\end{tabular}

PARITY

\begin{tabular}{llllll}
\hline $\mathbf{0}$ & 1 & 1 & 1 & & 1 \\
\hline $\mathbf{1}$ & 1 & 1 & 5 & 1 & \\
\hline $\mathbf{2}$ & & 3 & 3 & 5 & \\
\hline $\mathbf{3}$ & & 1 & 6 & 6 & 2 \\
\hline $\mathbf{3}$ & & 1 & 6 & 4 & 4 \\
\hline
\end{tabular}

SEXUAL PARTNERS

\begin{tabular}{|c|c|c|c|c|c|}
\hline \multicolumn{6}{|l|}{ (2) } \\
\hline 1 & & 1 & 4 & 2 & 1 \\
\hline 2 & & 2 & 2 & 3 & \\
\hline 3 & 1 & 3 & 7 & 5 & 2 \\
\hline 4 & 1 & & 3 & 2 & \\
\hline$>4$ & & & 5 & 4 & 1 \\
\hline SMOKING & & 3 & 2 & 2 & \\
\hline Co-morbidity & 4 & 11 & 30 & 28 & 10 \\
\hline Sexual Barriers & 1 & & 2 & 3 & \\
\hline SINGLE & 2 & 7 & 20 & 13 & 6 \\
\hline MARRIED & & & 2 & 3 & \\
\hline
\end{tabular}

Figures 


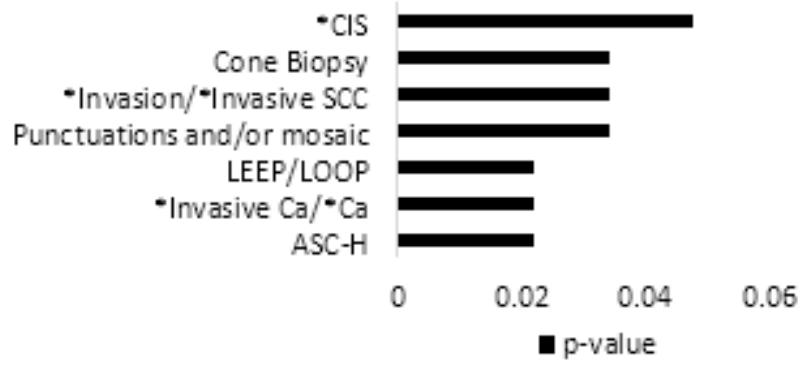

\section{Figure 1}

Multivariate Analysis by Linear Regression in Single Screening Modalities * - Suspected of/diagnosed with/cannot be excluded Carcinoma in situ (CIS), squamous cell carcinoma (SCC) Cancer (Ca), Atypical squamous cells high grade (ASC-H), Loop electrosurgical excision procedure (LEEP/LOOP)

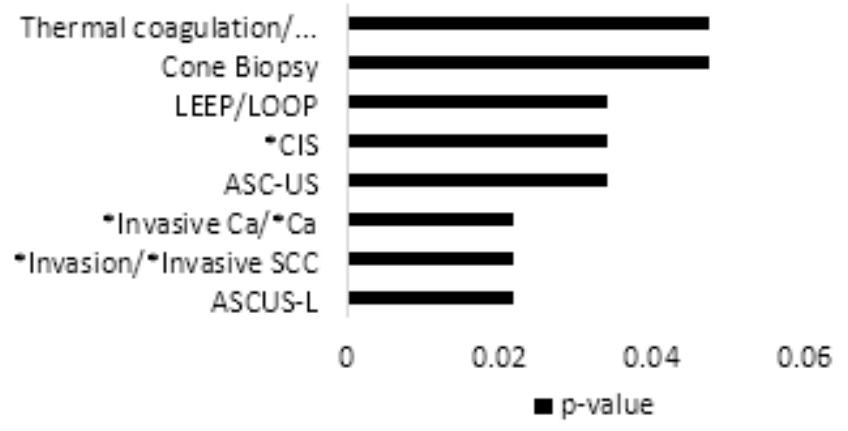

\section{Figure 2}

Multivariate Analysis by Linear Regression in Combined Screening Modalities * - Suspected of/diagnosed with/cannot be excluded Carcinoma in situ (CIS), squamous cell carcinoma (SCC) Cancer (Ca), Atypical squamous cells high grade (ASC-H), Loop electrosurgical excision procedure (LEEP/LOOP) Atypical squamous cells of undetermined significance (ASC-US)

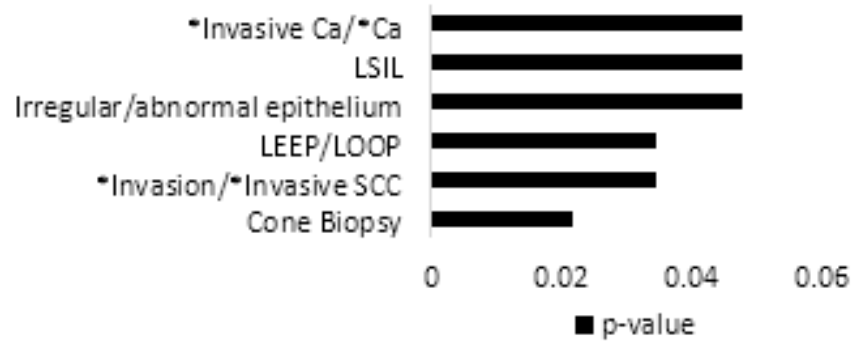

\section{Figure 3}

Multivariate Analysis by Linear Regression in Triage Screening Modalities * Suspected of/diagnosed with/cannot be excluded Carcinoma in situ (CIS), squamous cell carcinoma (SCC) Cancer (Ca), Atypical 
squamous cells high grade (ASC-H), Loop electrosurgical excision procedure (LEEP/LOOP). 